\title{
2-face viewable liquid crystal display using a 2-domain vertically aligned cell
}

\author{
Chao Ping Chen, ${ }^{* 1,2}$ Te-Chen Chung, ${ }^{2}$ and Tean-Sen Jen ${ }^{2}$ \\ ${ }^{I}$ Department of Electronic Engineering, Shanghai Jiao Tong University, Shanghai, China, \\ ${ }^{2}$ Product R\&D Center, Infovision Optoelectronics Co. Ltd., Kunshan, Jiangsu, China
}

Received February 14, 2011; accepted March 23, 2011; published March 31, 2011

\begin{abstract}
We propose a solution for obtaining a 2-face viewable liquid crystal display (LCD) that is able to show different images on the same screen depending on the viewing perspectives. Without requirement for any extra optical component, this function is simply realized by a 2-domain vertically aligned (VA) liquid crystal (LC) cell, which can accommodate two distinct viewing cones in its own right.
\end{abstract}

For many years, the display industry has placed too much emphasis in widening the viewing angle of LCD monitors and the like. But for some newly emerging applications, e.g. viewing angle switchable [1]-[4] or multi-view LCDs [5], wide viewing angles are no longer necessary. Instead, localized and split viewing angles are desired. The parallax barrier [6], adopted in Sharp's proprietary two-way viewing-angle LCD, and lenticular lenses [7] are the commonly used physical means to route light from subpixels to each independent field of view. However, both of them indeed demand an add-on device to assist a major LCD panel in order to fulfill their function. This will inevitably be accompanied by a sophisticated process of production, resulting in a decrease in the yield. Besides, a noticeably raised cost due to the parallax barrier or lenticular lens would not appeal to many manufacturers and consumers. Another approach to achieving multiple viewing angles is known as the vector error diffusion, a halftoning method [8]. Since this method involves signal processing in imaging, the output multi-view effect is very limited and problematic. Motivated by the above issues, we propose a solution to obtain easily a 2 -face viewable LCD that is able to show different images on the same screen, depending on the viewing perspectives.

Figure 1 schematically depicts the suggested structure, which is characterized by a 2-domain vertically aligned (VA) cell. As the alignment directions both on the upper and lower substrates and in the adjacent two domains are disposed anti-parallel, LC directors will correspondingly tilt in opposite ways upon the application of voltage, as shown in Fig. 1(b). To form a 2-domain structure, patterned electrode [9], ion beam alignment [10] and photo

*E-mail: rockreeves@hotmail.com alignment [11] have been known so far as adequate methods.

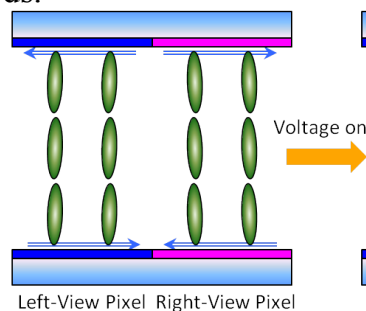

(a)

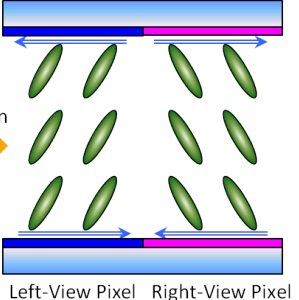

(b)
Fig. 1. Structure of a 2-domain VA LC cell when voltage is switched (a) on and (b) off.

In optics, the optical transmittance of a homogeneously aligned nematic LC layer between crossed polarizers is formulated as [1]:

$$
T=\sin ^{2}(2 \phi) \sin ^{2}\left(\frac{\Gamma}{2}\right)
$$

where $\phi$ is the angle between the LC director and the transmission axis (TA) of the analyzer, and $\Gamma$ is the phase retardation,

$$
\begin{gathered}
\Gamma=\frac{2 \pi\left(n_{e}(\theta)-n_{o}\right) d}{\lambda}, \\
n_{e}(\theta)=\frac{n_{o} n_{e}}{\sqrt{n_{o}^{2} \sin ^{2} \theta+n_{e}^{2} \cos ^{2} \theta}} .
\end{gathered}
$$

Here, $\lambda$ is the wavelength of the incident light, $\theta$ is the angle between the LC director and the wave vector of incident light, $d$ is the thickness of the LC layer, and $n_{e}$ and $n_{o}$ are the extraordinary and ordinary refractive indices of the nematic LC, respectively. In Eq. (1), if we fix the angle $\phi$ at $\pi / 4$, the transmittance can be considered as a function of the retardation $\Gamma$ of the $L C$ layer alone.

To understand its operational principle, we shall first, without preference, focus on the viewing angle variations of a right-view pixel, since the same thing happens to the other half as well. Referring to Fig. 2(a), the director of LC is initially aligned almost homeotropic to the substrate, considering a pretilt angle of nearly $90^{\circ}$. According to Eq. (1), a perfect dark state, or the minimum transmittance, owing to $\theta=0^{\circ}$ would be obtained at the normal direction, 
accompanied by two imperfect dark states situated side by side. When the director of LC is rotated to a certain angle in a counter-clockwise fashion, as shown in Fig. 2(b), the dark state will be shifted to the above angle, at which the incident light is in line with the LC director, making $\theta=0^{\circ}$. On the other hand, a perfect bright state, or the maximum transmittance, will be obtained at the other side as the transmittance gradually increases from the left to the right. At the final stage (see Fig. 2(c)), the LC director will be rotated till it is parallel to the substrate. Under this case, the bright state is shifted to the normal and the transmittance becomes symmetric about the normal. If only the transmittance variation from Fig. 2(a) to 2(b) is used for display, we could expect a right-facing viewing cone, which displays information towards the right side. Combined with that of the left-view pixel addressed simultaneously, we are therefore able to realize the so-called 2-face viewable display.

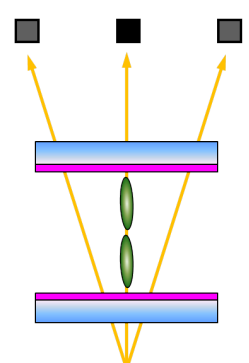

(a)

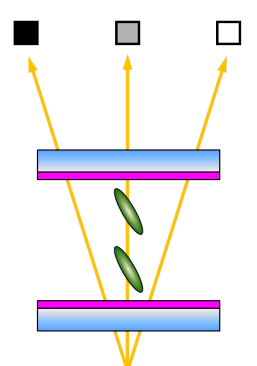

(b)

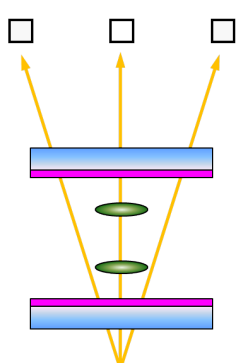

(c)
Fig. 2. Operational principle of 2-face viewable VA cell.

Based on the foregoing principle, we perform a numerical simulation on the platform of LCD Master with the following cell parameters: LC: MLC-6608 (Merck), $\Delta \mathrm{n}=0.083, \Delta \varepsilon=4.2, d=3.65 \mu \mathrm{m}$, and a pretilt angle of $89^{\circ}$. TAs of two crossed polarizers are set at $45^{\circ}$ relative to the LC's alignment direction. We shall mention that the viewing angle is calculated merely with respect to the right-view pixel, while the left one is omitted for the sake of clarity. Figure 3 shows the calculated voltage-transmittance $(\mathrm{V}-\mathrm{T})$ curves for three polar angles of $0^{\circ},+30^{\circ}$ (right), and $-30^{\circ}$ (left). In the voltage regime from 0 to $3.2 \mathrm{~V}$, a right-face viewable mode can be obtained as the transmittance of the left side always stays at a lower level than that of the right side. This means that an observer's eyes would be more focused to the right-view pixel rather than the left. If the left-view pixel is also being active next to the right-view pixel and addressed with different signals, the so-called 2-face viewable mode can be thus realized. The contrast ratio (CR) for the right-view pixel is about 17:1 (White state: $3.2 \mathrm{~V} /$ dark state: 0V). For achieving better CRs, we recommend using wide-viewing-angle polarizers to suppress light leakage from the bisector of crossed polarizers [12].
In order to quantitatively evaluate the crosstalk [13] for this two-view system, we shall define both static and dynamic crosstalk as:

$$
\begin{aligned}
& \text { Static Crosstalk Ratio }(\%)=\frac{T_{L}}{T_{R}}, \\
& \text { and } \quad \text { Dynamic Crosstalk Ratio }(\%)=\frac{\overline{T_{L}}}{T_{R}} .
\end{aligned}
$$

$\mathrm{T}_{\mathrm{L}}$ : Transmittance of the left side;

$\mathrm{T}_{\mathrm{R}}$ : Transmittance of the right side;

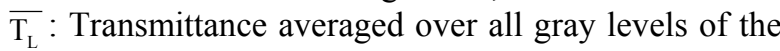
left side.

The calculated results of crosstalk ratios are given in Fig. 4, where both static and dynamic crosstalk are found relatively low in the high voltage regime. Therefore, it is reasonable to lessen the crosstalk at the cost of CR by offsetting the dark state voltage to a higher value. Another way is to properly dispose the black matrix in the inter-pixel region.

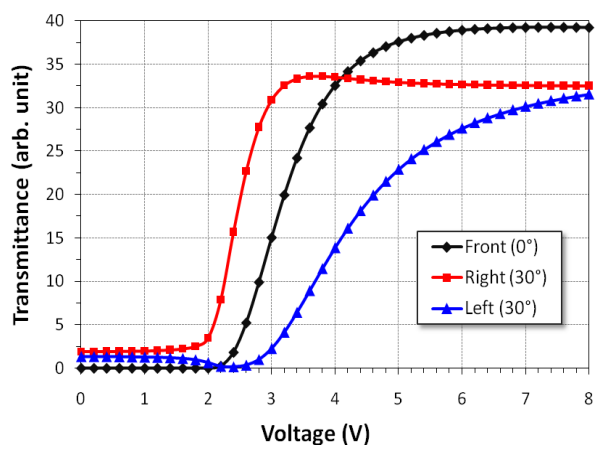

Fig. 3. Calculated V-T curves of 2-face viewable VA cell for three polar angles of (a) $0^{\circ}$, (b) $+30^{\circ}$, and (c) $-30^{\circ}$.

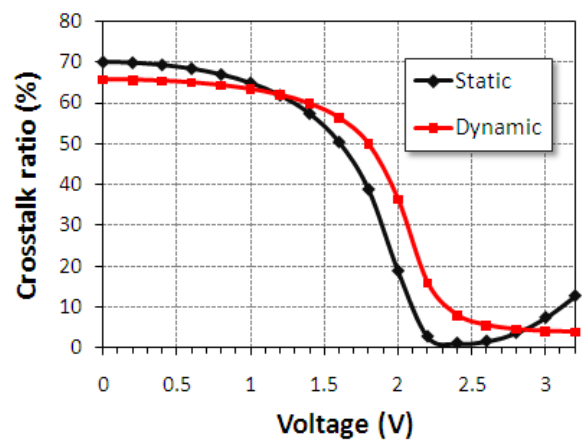

Fig. 4. Calculated static and dynamic crosstalk ratios.

The iso-luminance contour is plotted in Fig. 5, where the right-view pixel is turned on at $3.2 \mathrm{~V}$. It can be seen that the outcoming light is mainly directed towards the right direction. In addition, the iso-CR contour is given in Fig. 6, where the high levels of CRs are localized to the right half of the viewing cone, which agrees with our previous 
discussion. The best viewing condition for the right-view pixel shall be observed nearby $30^{\circ}$ in order to guarantee both high luminance and CR. Incidentally, we have also found out that the usage of an E-type polarizer [14] could yield better viewing angle characteristics and CRs than an O-type polarizer, even though it is at the cost of a significant loss of light in the off-axis area.

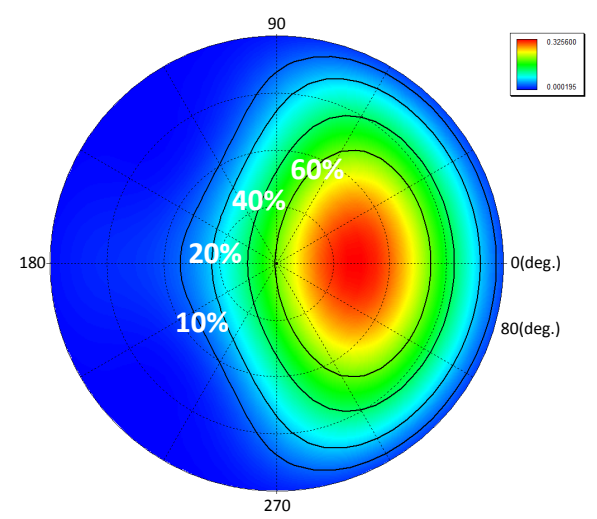

Fig. 5. Calculated iso-luminance contour at $3.2 \mathrm{~V}$.

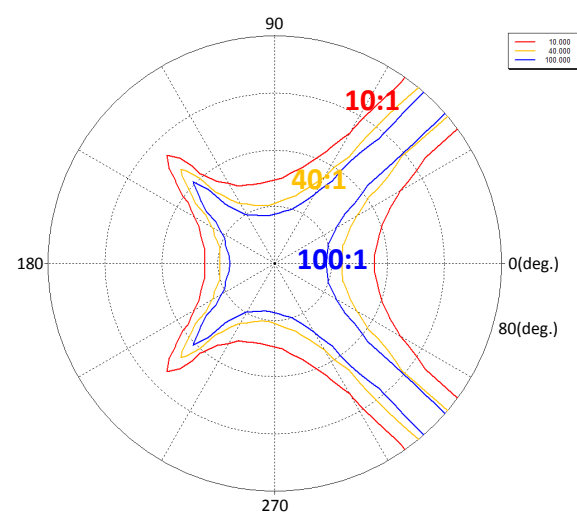

Fig. 6. Calculated iso-CR contour (3.2 V: $0 \mathrm{~V})$.

In experiments, we fabricated a 2-domain VA cell using the same parameters as the simulation. To provide opposite alignment directions in neighboring domains, a traditional rubbing process with a patterned mask was made to our cell. To simply exhibit different contents from left and right sides, the top electrode is common, whereas the bottom electrode is etched out with desired patterns. Figure 7 shows photos, which are taken from various angles, of our 2-face viewable VA cell, with the left face displaying the letter $\mathrm{H}$, while the right face is displaying number 2 . The pronounced crosstalk effect is inevitable in that the pitch of our fabricated cell is not fine enough to seamlessly overlay two images of right-view and left-view pixels. Finally, we shall notice that, for a given screen resolution, the two subpixels will divide the effective resolution in the horizontal direction. By way of example, if the total resolution of a display is $800 \times 480$, each viewable image would be $400 \times 480$.

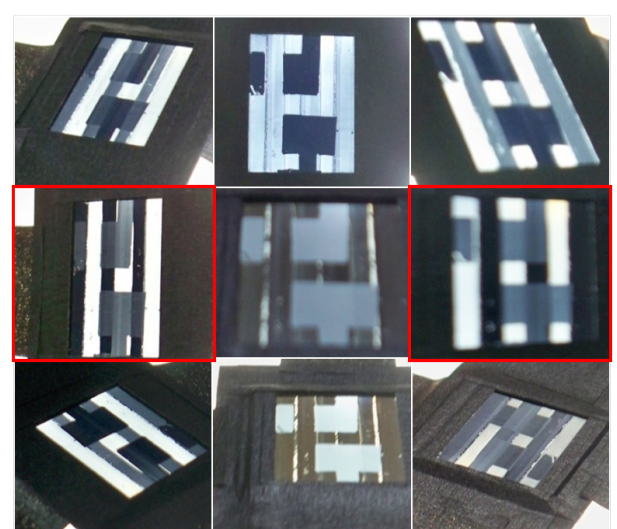

Fig. 7. Photos of the fabricated 2-face viewable VA cell taken from various angles.

In conclusion, we have demonstrated a 2-face viewable LCD device that is capable of allowing simultaneous displays of two independent image or video contents on the same screen. The proposed structure features a 2-domain VA cell, which has hence two different viewing cones. We are looking forward to its commercial potential in the diverse market of display not only for its versatile functions but also for its competitive manufacturing cost.

The authors would like to acknowledge Dr. Jae Chang Kim (Pusan National University) for his unfailing enlightenment.

\section{References}

[1] C.P. Chen, C.G. Jhun, T.-H. Yoon, J.C. Kim, Jpn. J. Appl. Phys. 46, L676 (2007).

[2] C.P. Chen, C.G. Jhun, T.-H. Yoon, J.C. Kim, Opt. Exp. 15, 17937 (2007).

[3] J.-I. Baek, Y.-H. Kwon, J.C. Kim, T.-H. Yoon, Appl. Phys. Lett. 90, 101104 (2007).

[4] C.P. Chen, K.-H. Kim, T.-H. Yoon, J.C. Kim, Jpn. J. Appl. Phys. 48, 062401 (2009).

[5] C.P. Chen, J.H. Lee, T.-H. Yoon, J.C. Kim, Opt. Lett. 34, 2222 (2009).

[6] D.J. Montgomery, M. Khazova, US patent 6859256 (2005).

[7] H. Ren, S.-T. Wu, Opt. Exp. 14, 11292 (2006).

[8] C.W. Wu, IEEE Signal Proc. Lett. 10, 225 (2003).

[9] Y. Qiao, T.-C. Chung, X. Huang, L. Zhou, Y.-W. Chiu, C.-T. Liao, T.-S. Jen, D. Zhang, SID Symp. Dig. Tech. Pap. 41, 830 (2010).

[10] P.K. Son, B.K. Jo, J.C. Kim, T.-H. Yoon, S.J. Rho, S.T. Shin, J.S. Kim, S.K. Lim, J.H. Souk, Jpn. J. Appl. Phys. 47, 8476 (2008).

[11] K. Hanaoka, Y. Nakanishi, Y. Inoue, S. Tanuma, Y. Koike, K. Okamoto, SID Symp. Dig. Tech. Pap. 35, 1200 (2004).

[12] T. Ishinabe, T. Miyashita, T. Uchida, Jpn. J. Appl. Phys. 41, 4553 (2002).

[13] Y.-J. Wu, Y.-S. Jeng, P.-C. Yeh, C.-J. Hu, W.-M. Huang, SID Symp. Dig. Tech. Pap. 39, 260 (2008).

[14] V. Chigrinov, E. Prudnikova, K. W. Ng, and H. S. Kwok, Jpn. J. Appl. Phys. 42, 1297 (2003). 